\title{
La enseñanza basada en antecedentes socioculturales de los alumnos: un método de eliminación de la desigualdad para lograr el aprendizaje significativo
}

\author{
Hatice Ezgi Aktuna y María Luisa Oliveras. Universidad de Granada \\ Recepción: 8 de junio de 2016 | Revisión: 10 de junio de 2016 | Aceptado: 20 junio de 2016 \\ Correspondencia: oliveras@ugr.es \\ Citar: Aktuna, H.E. y Oliveras, M.L. (2016). La enseñanza basada en antecedentes socioculturales de los alumnos: un método de \\ eliminación de la desigualdad para lograr el aprendizaje significativo. ReiDoCrea, 5, 130-145.
}

\begin{abstract}
Resumen: El propósito de este estudio es mostrar los resultados de una propuesta de enseñanza basada en antecedentes socioculturales de los alumnos a través de actividades culturalmente relevantes extraídas de sus prácticas cotidianas, investigando la contribución de esta propuesta a las actitudes y aprendizajes de los estudiantes sobre el concepto de medición de la magnitud área. Los datos fueron recogidos de 12 estudiantes de sexto grado (1112 años), en una escuela pública rural ubicada en Esmirna (Turquía). El diseño de la investigación fue interpretativo básico y de investigación-acción, ya que la investigadora fue profesora de la clase al mismo tiempo. Las observaciones de clases, notas de campo, grabaciones de vídeo, hojas de tareas de estudiantes, y los formularios de evaluación de las actividades se utilizaron para evaluar las percepciones de los estudiantes y su aprovechamiento. Los datos obtenidos se describieron en detalle y se analizaron de forma cualitativa y cuantitativa, (Aktuna, \& Çakıroğlu, 2013). Los resultados del estudio revelaron que una enseñanza basada en la realización de actividades fundamentadas en las prácticas laborales propias del entorno social, del sector del olivar, produjo un aumento de la motivación, el interés y confianza en sí mismos de los estudiantes; comodidad y disfrute en las clases de matemáticas, y la promoción de su propia cultura en el aula de matemáticas. Se observaron los cambios más positivos en los estudiantes de rendimiento medio y medio-bajo mientras que los estudiantes de bajo y alto rendimientos mantuvieron su estado habitual de motivación, actividad y nivel de aprendizaje.
\end{abstract}

Palabras clave: Medición de área | Etnomatemáticas

Teaching based on sociocultural background of the students: A method of eliminating inequality to achieve meaningful learning

\begin{abstract}
The purpose of this study is to show the results of a teaching proposal based on socio-cultural background of students through culturally relevant activities selected from their daily practices; by investigating the contribution of this proposal to the attitudes and learning of students about the area measurement concept. Data was collected from 12 sixth graders (11-12 years), in a rural public school in Izmir (Turkey). The research design was basic interpretative and action research because the researcher was the teacher of the class at the same time. The classroom observations, field notes, video recordings, student sheets and evaluation forms of activities were used to assess the perceptions of students and their improvement. The obtained data is described in detail and analyzed qualitatively and quantitatively (Aktuna, \& Cakiroglu, 2013). The study results revealed that teaching based on activities from labor practices of the social environment of the olive sector increased motivation, interest and self-confidence of the students; comfort, enjoyment, and promotion of their own culture in the mathematics classroom. The most positive changes in student performance were observed on middle and lower-middle achievers, while low and high achievers maintained their usual state of motivation, activity and level of learning.
\end{abstract}

Keywords: Area Measurement | Ethnomathematics

\section{Introducción}

En las sociedades industrializadas, una diferencia considerable en el rendimiento podría ser fácilmente observada entre los estudiantes que provienen de clases socioeconómicas más bajas y de origenes étnicos desfavorecidos y los estudiantes que provienen de la clase media y origen étnico dominante. Se ha argumentado que esta diferencia es bastante obvia porque las necesidades sociales y culturales de los estudiantes en desventaja no se cumplen, no pueden adaptarse suficientemente a los procesos educativos, debido a sus orígenes y también a su situación de desventaja que se produce por varios factores en el aula (el poder, las relaciones, actitud docente, currículo, etc.); por lo que la brecha en el rendimiento entre los estudiantes siempre sigue existiendo (Oakes, 1990; Secada, 1992; Tate, 1997). De esta manera, la importancia de los factores sociales, culturales y económicos en la educación no puede ser negada y forma parte del derecho a la propıa ımágen, en el momento en 
que nos damos cuenta de las razones por las cuales algunos estudiantes tienen éxito mientras que los otros no lo tienen. En tal sistema, los estudiantes de las minorías étnicas y de clase trabajadora tienden a quedarse atrás en la jornada académica (Civil, 2002).

Equivocadamente, la educación matemática y científica está generalmente etiquetada cómo un campo independiente de la cultura y que sólo contiene: cálculos numéricos, aplicación de teorías o ecuaciones, la transmisión de conocimientos técnicos, etc.

Esta idea, que hace caso omiso de que las matemáticas son un producto humano que refleja el conocimiento cultural tanto como otros campos, es también sorprendentemente muy común no sólo en la sociedad, sino también en las clases de matemáticas. Los maestros y los estudiantes, en su mayoría, no son conscientes de la conexión entre las matemáticas y la cultura, debido a la ausencia de la cultura en el contenido y la instrucción en estas aulas tradicionales (D'Ambrosio, 2001; Bishop, 2002). Sin embargo, en contraste con esta idea errónea hay un área emergente de estudio, llamada etnomatemáticas (Oliveras, 2006), que considera los aspectos socioculturales de las matemáticas en el aula y acepta los antecedentes culturales de los estudiantes como un recurso para vincularse con el contexto en las actividades matemáticas (Fasheh, 1997a).

Las Etnomatemáticas se encuentran aquí como un campo para eliminar los problemas de desigualdad, y tienen procesos educativos más justos. En contraste con la idea común, el campo de la educación matemática merece poner mucha más atención sobre los problemas de desigualdad, debido a su función de guardián en la sociedad. Las Matemáticas sirven como un "filtro crítico" en la sociedad, con su potencial para premiar a los estudiantes exitosos con un alto estatus ocupacional y salarial (Campbell, 1991). En otras palabras, los estudiantes tienen que alcanzar las matemáticas para ganar lugares en otros estudios académicos, de gran prestigio y posiciones bien pagadas (Meaney, 2002). Las matemáticas no sólo sirven como una escalera de movilidad económica, sino que también son esenciales para hacer que los consumidores y los votantes estén informados. Por otra parte, la competencia matemática es una clave en la lucha por la igualdad racial (Moses, 1994).

Sin embargo, las matemáticas suelen ser la disciplina con más fracasos en escuelas primarias y secundarias en Turquía (Tiraş, 1999). Además, Turquía es uno de los países de la OCDE con más disparidad inter-escolar en matemáticas, según PISA 2003 (EARGED, 2005). En resumen para no dejar que los niños se estanquen en las matemáticas y las ciencias, hay que darles el tratamiento completo para la educación matemática y sus dimensiones sociales. Por lo tanto, este estudio está conformado con un punto de vista etnomatemático, para ser propuesto como un método de eliminación de la desigualdad para alcanzar y lograr la comprensión matemática más significativa, relacionada con los antecedentes culturales y sociales de los alumnos.

1.1 Propósito del estudio: Investigar las percepciones de los estudiantes de sexto grado y sus compromisos en las tareas matemáticas relacionadas con el concepto de medición de la magnitud área, introduciendo modificaciones en la metodología de la clase, basadas en las prácticas profesionales de los trabajadores del sector del olivar.

1.2 Pregunta de Investigación: ¿Cómo perciben y colaboran los estudiantes con las tareas de medición, al introducir una metodología docente enriquecida con etnomatemáticas? 
1.3 Importancia del estudio: Cuando se aplican enfoques etnomatemáticos, las matemáticas de la escuela son cada vez más relevantes y significativas para los estudiantes y también promueven la calidad general de la educación (Adam, 2004). Los estudiantes se sienten más cómodos y seguros sobre la discusión de los conceptos matemáticos y obtienen una mejor apreciación de las matemáticas, cuando se les enseña con una perspectiva cultural (Schultes y Shannon, 1997). Por ello, este estudio trata de ofrecer una metodología y unas actividades fundamentadas en etnomatemáticas, relacionadas con la particularidad cultural de los estudiantes, cómo una estrategia de enseñanza científico-matemática alternativa, para que la reciban de una manera significativa y comprensible.

En sociedades diversas, podemos ver que la cultura experimentada por los estudiantes en sus hogares rara vez es la misma que la representada por el plan de estudios (Bishop, 1994). Esto revela que, para muchos niños en todo el mundo, las experiencias matemáticas en la escuela no están culturalmente en consonancia con sus experiencias en casa (Bishop, 2002). Este desajuste es uno de los factores que contribuyen al bajo rendimiento de los estudiantes que provienen de subculturas (Lubienski, 2001). En Turquía, por ejemplo, un país que cuenta con una amplia diversidad cultural en términos de clase social, geografía, origen étnico, idioma y religión; no podemos ignorar los problemas de los estudiantes que están expuestos a un plan de estudios que no respeta dichos factores culturales. En el contexto de Turquía, la educación es uniforme para todos los grados en todas las escuelas y en todas las regiones del país, con un plan de estudios a nivel nacional que fue preparado por el Ministerio de Educación Nacional. La transmisión y el avance de la cultura dominante turca es una parte integral de esta educación. La presencia de cualquiera de las sub-sociedades y subculturas, su existencia histórica, sus lenguas, valores, normas y formas de vida son ignorados en el sistema escolar formal (Sahin y Gulmez, 2000). Desafortunadamente, esas cuestiones no atraen suficiente atención en el campo de la educación, en especial la educación matemática en Turquía. Sin embargo, casi una cuarta parte de la población de niños desde los 8 a los 15 años son pertenecientes a grupos étnicos minoritarios, y existen notables diferencias en los resultados educativos a través de estos grupos (kurdos, árabes, caucásicos) (Kirdar, 2009).

Como las etnomatemáticas y los aspectos sociales de la educación matemática casi no se han discutido todavía allí, cualquier investigación relacionada con los métodos y la integración de tareas sobre etnomatemáticas parecen ser valiosas como punto de partida. Con este sentido, este estudio podría ofrecer buenas prácticas para nuestro país, y para otros países donde las circunstancias sociológicas y educativas sean semejantes, con el fin de permitir que los niños aprendan matemáticas para solucionar sus necesidades dependiendo de su cultura.

\section{Literatura}

Bishop (1988a) distingue entre las matemáticas con una $M$ mayúscula y con una pequeña $\mathrm{m}$. M es la matemática aceptada como disciplina científica e institucional aplicada por los matemáticos y estudiantes de ingeniería o física, altamente especializados, sobre todo en las universidades. Sin embargo, $\mathrm{m}$ se determina como los procedimientos que una persona utiliza en su vida diaria, tales como contar y medir. A partir de esta distinción, François y Pinxten (2007) se preguntan si "debemos aceptar la idea de que $\mathrm{M}$ del matemático deba ser aprendida por todos, o debemos desarrollar el $\mathrm{m}$ en la cultura de los sujetos a través de nuestras clases de matemáticas" (pp. 214). 
Las Etnomatemáticas (Oliveras, 2006), que es una de las teorías naturalistas sobre el conocimiento matemático, se forma como una alternativa a los puntos de vista absolutistas como el formalismo, el logicismo y el constructivismo (Ernest, 1991). Las Etnomatemáticas y otros defensores del enfoque naturalista proponen que el conocimiento matemático (incluyendo reflexiones y sentimientos de $\mathrm{M}$ ) tiene raíces culturales y contextuales en la naturaleza (Pinxten, 1992). En este estudio, se acepta la perspectiva naturalista de las matemáticas.

Como en todas las culturas del mundo se desarrollan actividades de maneras particulares, también necesitan ejercicios específicos para aprender, para hacer referencia a sus habilidades matemáticas (Presmeg, 1998). Si aceptamos que los individuos y las culturas desempeñan un papel activo en el aprendizaje y la producción de las matemáticas, es normal que se rechacen los enfoques de enseñanza que tengan en cuenta las matemáticas como un cuerpo de conocimiento descubierto. Por lo tanto, las matemáticas exigen un enfoque multicultural que mejora la conciencia de los estudiantes y el respeto a sí mismos y a los demás al exponerlos a las matemáticas de diferentes culturas (İzmirli, 2011).

\subsection{Por qué el tema de medición?}

Bishop (1991) afirma que las matemáticas se producen en todas las culturas en seis aspectos de la actividad humana. Esas categorías en las que se produce la actividad matemática son: contando, midiendo, localizando, diseñando, interpretando y explicando. La categoría de medición se identifica como; "La medición es la tercera universal y significativa actividad para el desarrollo de las ideas matemáticas, y se refiere a la comparación, con orden y con cuantificación de cualidades, factores que le dan el valor y la importancia. Todas las culturas reconocen la importancia de ciertas cosas, pero una vez más, todas las culturas no valoran las mismas cosas en la misma medida. Mucho depende del entorno local y las necesidades que éste provoca" (pp. 34). Para este punto de vista, la medición como una de las principales actividades del ser humano parece ser un buen punto de partida para desarrollar y aplicar actividades de matemáticas culturalmente relevantes en un aula de primaria.

La medición es también una actividad matemática que todas las culturas tienen que utilizar (Bishop, 1988a), pero de diferentes maneras, métodos y enfoques. Sin embargo, las diferentes prácticas culturales entre la matemática escolar y familiar en su mayoría causan conflictos para los estudiantes. En el estudio de Bishop (1978), el entrevistado, de Papúa Nueva Guinea, reveló su conflicto en la búsqueda de área en diferentes contextos socioculturales, diciendo que "En casa sumo, en la escuela multiplico". Bishop explica la importancia de tener en cuenta el efecto de los valores culturales de los conceptos de medición con el ejemplo de algunos aldeanos que miden el tamaño de un jardín por el número de pasos de anchura y longitud del jardín, incluso si los occidentales piensan que es un semi-perímetro. Sin embargo, en la escuela, el mismo estudiante calculará el área de la zona como un producto de la longitud y la anchura, que demuestra claramente que los niños de diferentes antecedentes pueden tener diferentes perspectivas en las variables de la zona.

Del mismo modo, en el estudio de Owens y Kaleva (2007) en Australia, los estudiantes estaban pensando intuitivamente la superficie en términos de longitud, pero no como un producto de dos lados como se presenta en la matemática escolar, ni como un semi-perímetro sino como una manera de contar las áreas fijas conocidas de su contexto. Esta variedad sobre los estilos de medición nos dio perspectivas para conectar nuestro discurso en la actividad de medición en el aula, al cuestionar cómo todas las sociedades comparan y miden sistemáticamente. En realidad, requiere de 
habilidades especiales de los educadores matemáticos y hablantes nativos para descubrir los sistemas de medición existentes y convertirlos en las actividades a realizar en el aula (Bishop, 1988a).

Además de todo, la medición es un tema bastante difícil para los estudiantes de todo el mundo (Zacharos, 2006). Los conceptos de Area y Perímetro, reconocer la estructura de los sistemas de medición y su estimación, son temas que a menudo no logran comprender los estudiantes (Kaleva, Matang y Owens, 2008). Del mismo modo, en Turquía, los estudiantes tienen dificultades en la alineación con una regla, en distinguir el perímetro del área, unidades de longitud y de medición de área (TanSisman y Aksu, 2011). Al darnos cuenta de la importancia y la dificultad del tema de medición, decidimos utilizar el enfoque etnomatemático en la unidad didáctica de medida, porque de acuerdo a la literatura, se sabe que las comunidades tradicionales son bastante fuertes en las habilidades de medición, así que quisimos tomar su ventaja como fuente de inspiración en las clases de matemáticas.

Podemos presentar muchos otros estudios que revelan los efectos positivos del uso de la cultura o de las experiencias culturales extraescolares de los estudiantes para la medición, en lugar de sugerir libros de texto monotipo; tales como: "unidad etnomatemática de medición" (área, perímetro, volumen) que fue diseñado por Adam (2004) sobre los sistemas de medición en la cultura de Maldivas para estudiantes de $5^{\circ}$ grado. El enfoque etnomatemático fue apreciado y entendido por los profesores y estudiantes. Los estudiantes ganaron motivación e interés, vieron el funcionamiento de las matemáticas en el trabajo y en la sociedad, las matemáticas conectadas a las actividades del mundo real. Masingila (1993) pasó un verano con un grupo de colocadores de alfombras para proponer una tarea de medición etnomatemática de $6^{\circ}$, $7^{\circ}$ y $8^{\circ}$ grado sobre la realización de los conceptos matemáticos y procesos que intervienen en la estimación y la instalación de revestimientos para el suelo y tuvo resultados positivos similares. Aunque no lo tuvimos en cuenta, el estudio de las artesanías realizado por Oliveras (1996) muestra la medida en tres niveles: micro (taracea), medio (alfombras) y macro (empedrados), y su aplicación a la enseñanza en España, con buenos resultados. El estudio de Kaleva, Matang y Owens (2007), que trata de sistemas de medición culturales de los diferentes grupos lingüísticos de Papúa Nueva Guinea, demostró que el enfoque cultural informal para la medición permite a los estudiantes comprender más fácilmente el significado de medición y cómo se estructuran las unidades.

\section{Metodología}

\subsection{Enfoque de la Investigación}

Este es un estudio interpretativo básico que se utiliza cuando un instructor está interesado en cómo los estudiantes interpretan una situación o fenómeno. En el estudio cualitativo basico, los datos se obtuvieron de entrevistas, observaciones o análisis de documentos (por ejemplo, el trabajo escrito de los estudiantes). El análisis es de patrones o temas comunes y el resultado es un profundo relato descriptivo que hace referencia a la literatura que ayudó a enmarcar el estudio (Merriam, 2002).

\subsection{Los participantes del estudio}

El estudio se realizó en una escuela pública en un pueblo de Kemalpaşa, Esmirna (Turquía). Los participantes eran 12 estudiantes de sexto grado, cuatro niñas y ocho niños, que vivían en el pueblo, donde la economía se basa principalmente en el cultivo del olivar. Este grupo de estudio fue seleccionado por conveniencia, ya que la 
investigadora fue profesora de matemáticas en esta escuela y su cultura particular era adecuada para la aplicación de un método etnomatemático. De acuerdo con los resultados de los exámenes anteriores y actuaciones en el aula, y la opinión de su maestro principal, la clase era heterogénea en términos de rendimiento en matemáticas.

\subsection{La propuesta didáctica elaborada e implementada}

En ese proyecto, siete nuevas actividades fundamentadas en las etnomatemáticas del olivar y otros aspectos culturales se han desorrollado por la investigadora. El tema "medición de área" fue explicado en el aula con esas tareas etnomatemáticas desarrolladas por la investigadora.

Antes de que el proceso de construcción de tareas fuese realizado, se revisó la literatura relacionada. Especialmente se analizaron los estudios que consisten en la aplicación práctica en detalle de los elementos etnomatemáticos. Los posibles temas futuros sobre medicion se observaron, para integrar en tareas etnomatematicas. Esta proceso no sólo proporcionó ideas creativas, inspiradoras y adecuadas sobre las tareas futuras, sino que también nos guió sobre el proceso de desarrollo de las tareas.

Con el objetivo de recopilar y analizar la información cultural sobre los sistemas de medición de los aldeanos, hicimos entrevistas en profundidad con los estudiantes, los aldeanos y los maestros, los cuales son del pueblo, proceso etnográfico que facilitó información rica para ser utilizada como ejemplos en la planificación de las lecciones para su implantación en las clases .

Además de eso, la investigación general sobre nuestro tema principal del olivar realizada en Internet y recopilando material escrito fue otro recurso para el desarrollo de tareas, tales como la revisión acerca de la oliva en la mitología, la historia y la religión, cómo diseñar un jardín de olivos, información y estadísticas sobre los productos de la oliva y sus técnicas, etc. Los foros en los que los olivareros discuten acerca de sus prácticas, y diferentes tipos de arte (pinturas, decoraciones, etc.) que son inspirados o alimentadas desde de oliva también proporcionaron materiales visuales y de datos para utilizar en nuestras tareas.

La programación curricular de matemáticas de sexto grado de Educación Nacional de Turquía también se consultó para que fuera coherente con las actividades y ayudó en el proceso de desarrollo de tareas, en la selección de los posibles temas a ser integrados y la adaptación de los ejemplos y problemas existentes en nuestro contexto.

Las tareas fueron revisadas y actualizadas para ser utilizadas en el aula,por dos profesores de matemáticas con postgrado de acuerdo con sus puntos de vista. Finalmente, después de estar de acuerdo en su contenido y orden, se desarrollaron las tareas, que fueron bien aceptadas por los estudiantes, y desarrolladas con éxito, en general.

Aquí mostramos, en la siguiente tabla 1, las siete tareas etnomatemáticas diseñadas y desarrolladas por la investigadora, con los objetivos y programacion temporal de la actividad. 


\begin{tabular}{|c|c|c|}
\hline Aetivities & Objectives & Duration \\
\hline Activity 1: Uncle Halil says that... & $\begin{array}{l}\text { Estimate area of plane regions by } \\
\text { using strategy }\end{array}$ & $22,23,29$ April \\
\hline Activity 2: While planting olives & $\begin{array}{l}\text { Explain area measurement units and } \\
\text { convert each other }\end{array}$ & 30 April;6, 7 May \\
\hline $\begin{array}{l}\text { Activity 3: Ancient measurement } \\
\text { systems \& measurement units in } \\
\text { Ottoman Empire }\end{array}$ & $\begin{array}{l}\text { Explain area measurement units and } \\
\text { convert each other }\end{array}$ & $13,14,20$ May \\
\hline $\begin{array}{l}\text { Activity 4: Rating the remaining } \\
\text { area of olive-tree }\end{array}$ & $\begin{array}{l}\text { Solve and build problems about the } \\
\text { area of plane regions }\end{array}$ & 21 May \\
\hline $\begin{array}{l}\text { Activity 5: The area of the land of } \\
\text { Nomads }\end{array}$ & $\begin{array}{l}\text { Solve and build problems about the } \\
\text { area of plane regions }\end{array}$ & 27 May \\
\hline $\begin{array}{l}\text { Activity 6: Olive and culture; } \\
\text { thousand years old mystical motif- } \\
\text { the tree of life }\end{array}$ & $\begin{array}{l}\text { Solve and build problems about the } \\
\text { area of plane regions }\end{array}$ & 28 May \\
\hline $\begin{array}{l}\text { Activity 7: The universal symbol of } \\
\text { peace- dove \& olive branch }\end{array}$ & $\begin{array}{l}\text { Solve and build problems about the } \\
\text { area of plane regions }\end{array}$ & 3 June \\
\hline
\end{tabular}

Tabla 1. Actividades usadas en la instrucción, los correspondientes objetivos y la programación temporal

Las hojas de tareas incluyen información preliminar, la actividad principal, intensificadores de ejercicios relacionados con las tareas, y los problemas que serán desarrollados. Como ejemplos se pueden ver la hoja de la tarea 4, en el anexo 1, y otra hoja de tarea 6 , en el anexo 2, en estas, al ser las originales, el texto se encuentra escrito en turco.

\subsection{Instrumentos y procedimiento para la recopilación de datos}

Con el fin de recopilar datos, se administraron diferentes tipos de instrumentos tales como grabaciones de vídeo, observaciones, notas de campo, hojas de tareas y formularios de evaluación de la actividad de los estudiantes durante y después de la instrucción.

Los datos se han recogido en el semestre de primavera del curso 2012 a 2013. La longitud de la instrucción excede el tiempo determinado para el tema de medición de la superficie en el plan anual, pero se debe considerar que, durante el proceso, además de las tareas etnomatemáticas, también tuvimos que cubrir todos los aspectos del plan de estudios nacional para las cuestiones éticas.

\subsection{Análisis de los datos}

El análisis de datos se inició con la etapa de reducción de datos propuesta por Miles y Huberman (1994) como la primera etapa de análisis de datos cualitativos, para decidir qué parte de los datos será útil de acuerdo con el propósito del estudio. Para el resto de los datos, adoptamos el método de análisis descriptivo, que requiere resumir e interpretar los datos obtenidos mediante diversas técnicas de recolección de datos. En 
este método de análisis, el investigador puede a menudo dar citas directas para reflejar los puntos de vista de los individuos observados o entrevistados. El propósito principal de este método de análisis de datos presenta los resultados para el lector de manera resumida e interpretada. Por lo tanto, durante el proceso de análisis de los datos de este estudio, los datos se leyeron y editaron sobre la base del marco teórico creado previamente, y fueron reunidos de una manera lógica. Entonces, los datos editados se describen haciendo referencia a las citas directas cuando sea necesario. Al final de este proceso, los resultados se dieron a conocer definidos, asociados e interpretados; y las relaciones de causa y efecto entre los resultados se explican en detalle a fin de fortalecer las interpretaciones (Yildirim y Simsek, 2003).

\section{Resultados y conclusiones}

\subsection{Las percepciones y compromiso de los estudiantes sobre las actividades etnomatematicas realizadas en concepto de la medicion de area}

Durante las actividades, se observó que los estudiantes participaron en las tareas relacionadas de acuerdo a sus conocimientos existentes obtenidos no desde la escuela, sino a partir de las prácticas diarias. Las tareas hablaban del concepto de medición, pero la mayor parte de los estudiantes, no importa su logro anterior, contribuyeron de alguna manera en su mayoría por compartir sus experiencias sobre el tema de medición en los olivares. Con esta actividad, los estudiantes comenzaron a ser más conscientes del hecho de que las matemáticas existen fuera de la escuela y en su cultura. Cuando a los estudiantes se les enseñó con contextos del olivar familiares, que ya se conocían inconscientemente, se sentían mejor y comenzaron a acertar también. Algunos de los estudiantes mencionaron que les gustaba porque se sentían más exitosos o que les resultó más fácil que anteriormente. Por lo tanto, cuando interpretamos las expresiones y la literatura de los estudiantes, se puede concluir que ganaron interés por las tareas matemáticas y luego la matemática se hizo significativa cuando se introdujeron las actividades familiares con métodos etnomatemáticos.

Los estudiantes generalmente disfrutaron y no se aburrieron durante las actividades integradas con etnomatemáticas (con algunas excepciones), estaban entusiasmados con los temas discutidos, completaron las tareas de una manera motivada y sin impacientarse y preguntaron acerca de la próxima actividad. Por otra parte, en los formularios de evaluación de la actividad, todos los estudiantes dijeron que prefieren seguir aprendiendo las matemáticas en la forma en que tuvieron durante el tema de medición de superficies, porque encontraron que era agradable, más fácil e interesante. Esto significa que la aplicación de las etnomatemáticas al plan de estudios fue apreciada por los estudiantes, aumentó su motivación e interés y preferían éste a otros métodos de matemáticas aplicados en clases anteriores. Además de aumentar la motivación y el interés, el estado de ánimo de los estudiantes fue notablemente diferente durante las actividades. Obviamente estaban teniendo un buen momento y trabajaron concentrados, motivados, enérgicos y sonrientes. Su comportamiento relajado en comparación con situaciones anteriores les llevó incluso a hacer chistes mediante el uso de conceptos matemáticos, cantar canciones y bailar mientras trabajaban en las tareas, hablaban, compartian y cooperaban con los demás. Este cambio de comportamiento y estado de ánimo, especialmente en los más tímidos y menos exitosos, parece ser el indicador de cómo los estudiantes estaban relajados y sin miedo; y cómo se puede obtener una mejor apreciación de las matemáticas cuando se les enseña desde una perspectiva cultural. 
Este interés obtenido de las actividades etnomatemáticas, parecía ser convertido en confianza en sí mismo de forma natural. Especialmente los que no son buenos en matemáticas pero habían tenido experiencia de primera mano en agricultura, daban respuestas más creativas y prácticas que las previstas durante las actividades. Ellos se dieron cuenta de que las prácticas que ya han estado aplicando estaban funcionando bien en esas tareas, de modo que se sentían seguros porque las tareas se han diseñado teniendo en cuenta las matemáticas de la vida real, pero la vida real de los agricultores aldeanos del olivar, no de las otras comunidades de la clase media. Además, su activo rendimiento y rápidas estrategias que desarrollaban para las situaciones, sin ningún tipo de orientación, hacía que pareciera que son dueños de los conocimientos, y que ese era su patio de recreo.

La segunda actividad, que tiene como objetivo enseñar a convertir unidades de medida en tareas de riego y fertilizado para el olivar, no despertó tanto interés como los otros y tenían una dificultad considerable. La razón probablemente fue incluir demasiada información técnica en la actividad, que se hacía difícil para ese grupo de edad. Además de eso la otra posible explicación para su falta de interés podría ser que el tema de esta actividad no se refería a las experiencias diarias de los estudiantes. La actividad fue sobre el cultivo de oliva pero, como se dijo antes, los estudiantes no fueron principalmente activos en el proceso de cultivo del olivo, sino en la cosecha. Deben al menos estar familiarizados, pero las prácticas de plantación de sus familias eran bastante diferentes a las indicadas en la actividad, ya que la contradecían durante las discusiones. Por lo tanto, cuando el tema integrado no coincide bien con las prácticas reales de los estudiantes, no pueden hacer la conexión y comienzan a aburrirse. Con esta observación, se puede concluir que esta actividad era débil para servir a los objetivos específicos del proyecto basado en la etnomatemática.

La otra actividad que no pudo influir en los estudiantes fue el del antiguo sistema de medición otomana. En esta actividad, se desarrolló con la suposición de que le iba a gustar, porque este antiguo sistema refleja su cultura desde las raices. Los estudiantes pensaron que esta actividad era irrelevante, inútil y llena de conceptos de medición complicados; estaba claro que se aburrieron y trataban de cambiar al tema de la oliva porque prefierian trabajar en él. Por eso, este hallazgo nos permite recordar que la verdadera cultura no es la que se tiene desde las raíces, sino los valores que creamos a través de nuestra existencia. Sin embargo, con esta actividad los estudiantes estaba expuestos a una tarea matemática que incluye muchas unidades de medida antiguas y en desuso, por lo que probablemente era una masa de expresiones sin sentido, en vez de una manera de entender su mundo. Este hallazgo fue identificado como una advertencia para el proceso de desarrollo de las nuevas actividades, tratamos por esto de reconocer las matemáticas en las prácticas culturales cotidianas de los estudiantes y de productos que puedan estar comprendidos en su mundo, en lugar de añadir en las actividades algunos elementos culturales acerca de su historia.

Se observa que cuando los estudiantes se presentan a las actividades basadas en pequeños problemas, se han concentrado en tareas con facilidad y motivación de forma contínua durante periodos de 2 horas. Además de sus preferencias acerca de las actividades menos largas, en los formularios de evaluación de la actividad se reveló claramente la deficiencia de las actividades largas. Por lo tanto, la longitud de las actividades podría ser un punto crítico para la concentración y el interés de los estudiantes, por lo que las actividades de gran longitud se deben evitar durante las actividades basadas en etnomatemáticas. Otro resultado observado durante las actividades fue que los estudiantes se integraban fácilmente en la resolución de problemas y seguían los pasos de manera adecuada y familiar. Por lo tanto, es natural que desarrollaran o mejoraran sus habilidades para resolver problemas con la ayuda de las actividades etnomatemáticas. Además, no se ha observado ningún problema en 
la lectura e interpretación de los problemas durante las actividades. Completaron fácilmente incluso los problemas difíciles siguiendo los pasos adecuados, porque probablemente conectaron y explicaron el significado mejor cuando se expusieron a un contexto familiar como el de la oliva.

El hallazgo más notable e interesante del estudio fue que los estudiantes de promedio medio y medio-bajo cuyo conocimiento y confianza en sí mismo estaban limitados, mostraron diferencias considerables en los medios de actuación y los comportamientos debido a las actividades etnomatemáticas; mientras que alumnos de rendimiento alto y bajo de la clase no mostraron diferencias de rendimiento visibles, en comparación con sus actuaciones anteriores. Aquellos estudiantes que en realidad tienen potencial para las matemáticas, pero se dan por vencidos o no saben cómo usarlo por algunas razones empezaron a obtener los conocimientos de nuevo con la ayuda de las actividades matemáticas atractivas, culturalmente pertinentes y familiares que ya sabían, por las circunstancias de su vida real. Los alumnos de alto rendimiento no tienen un punto de ruptura como los otros durante estas actividades porque ya estaban unidos e integrados a las matemáticas. Del mismo modo, los estudiantes con rendimiento bajo tampoco pudieron proporcionar datos considerables en la diferencia de su rendimiento. Probablemente porque no tienen suficientes bases matemáticas para dar un paso más allá de la participación en las clases de matemáticas. Por lo tanto, las actividades etnomatemáticas no jugaron un papel crítico en sus actuaciones, pero de alguna manera apreciaron y disfrutaron las actividades en general, y querían continuar las clases con actividades similares en el futuro.

Los que mejoraron, alumnos de rendimiento medio y medio-bajo, eran en realidad estudiantes social y culturalmente desfavorecidos, que tenían que trabajar en la agricultura para ayudar a sus familias y cuyas experiencias matemática en la escuela no están culturalmente en consonancia con sus experiencias en casa. Esta falta de coincidencia, probablemente, constituyó uno de los factores que contribuyen al bajo rendimiento de los estudiantes en matemáticas (Lubienski, 2001). Sin embargo, si se tiene en cuenta que los planes de estudios se construyen con los valores de la clase media y los triunfadores son generalmente los que estan bastante familiarizados con esos valores (Oakes, 1990; Secada 1992; Tate 1997), el aumento de rendimiento de los estudiantes en desventaja puede revelar que las situaciones problemáticas incluidas en las actividades parecían bastante relevantes para ellos culturalmente, y luego se dieron cuenta de su potencial para tratar con ellos. En medio de estas adquisiciones, en estas actividades integradas con etnomatemáticas era bastante interesante no dejar atrás a los estudiantes cumplidores de comprensión media-baja.

Creo que al utilizar el método etnomatemático, este estudio representa un pequeño, pero muy significativo paso para dar voz a la comunidad y mostrar el valor de los conocimientos de los agricultores locales, aldeanos del olivar.

En este estudio, el grupo objetivo no era los que son criados con valores urbanos de clase media, que era el grupo subcultural. De este modo se promovieron las prácticas y los valores diarios de los aldeanos durante las actividades y la población rural para regresarles el poder en el aula. De esta manera, creo que este estudio demostró que si el profesor promueve los valores propios de los estudiantes -incluso en las matemáticas, ya que están produciendo también el conocimiento matemático como práctica humana- en lugar de las que se presentan en los programas existentes, pueden exponer su verdadero potencial y pueden darles la oportunidad de tener éxito en las matemáticas. 
Además de los factores relacionados con las actuaciones de los estudiantes, otra adquisición positiva obtenida por este método se relaciona con el ambiente de la clase. De acuerdo con las observaciones, los estudiantes estaban siguiendo los pasos de las tareas y no dudaban de intercambiar conocimientos, discutir acerca de los conceptos y pedir uno al otro ayuda. Hubo un ambiente de intercambio comunicativo, productivo y emocionante en la clase condimentado con diversión y chistes. Fue agradable observar el entorno del aula porque cualquier tipo de conflicto social puede ser rechazado en este ambiente de clase y constituye una condición previa para un programa etnomatemático exitoso porque los estudiantes que comparten se sienten partícipes de sus actividades (Presmeg, 1988; Vithal y Skovsmose, 1997). Construyeron un ambiente de clase en el que todo el mundo respetó al otro y progresaron todos juntos. Como dijo uno de los estudiantes "Me sentí muy bien durante las actividades, porque la mayoría de los estudiantes eran capaces de resolver los problemas." Y añadió que se siente mal cuando otros no lo podían hacer. Esta idea probablemente fue el resultado de su preferencia por un ambiente solidario de clase, en lugar de uno agresivo.

Por lo tanto, hemos intentado presentar a los alumnos un ambiente cooperativo que les permite experimentar la comunicación y el intercambio durante las instrucciones y sus resultados positivos en las actuaciones se parecieron a los resultados coherentes de la literatura relacionada, que dice que un programa de matemáticas basado en la cultura altera, positivamente, la organización social y la comunicación en el aula (Lipka, 2005).

\section{Referencias}

Adam, S. (2004). Ethnomathematical ideas in the curriculum. Mathematics Education Research Journal, 16(2), 49-68.

Aktuna, E., \& Çakıroğlu, E. (2013), "Sixth grade students' perceptions of and engagement in ethnomathematical tasks in the area measurement concept". MA Thesis, Middle East Technical University, Ankara.

Bishop, A. (1978). Visualising and mathematics in a pre-technological culture. In E. Cohors-Fresenborg \& I. Wachsmuth (Eds.), Proceedings of the Second International Conference for the Psychology of Mathematics Education. (pp. 79-90). Osnabrück: PME.

Bishop, A.J. (1988a). Mathematics education in its cultural context. Educational Studies in Mathematics, 19(2), 179-191.

Bishop, A.J. (1991). Mathematical Enculturation. Dordrecht: Kluwer Academic Publishers.

Bishop, A.J. (1994). Cultural conflicts in mathematics education: developing a research agenda. For the Learning of Mathematics, 14(2), 15-18.

Bishop, A.J. (2002). Critical Challenges in Researching Cultural Issues in Mathematics Education. Journal of Intercultural Studies, 23(2), 119-131.

Campbell, P.B. (1991). So what do we do with the poor, non-white female? Issues of gender, race, and social class in mathematics and equity. Peabody Journal of Education, 66, 95-112.

Civil, M. (2002). Culture and Mathematics: a community approach. Journal of Intercultural Studies, 23(2), 133-148.

D'Ambrosio, U. (2001). What is Ethnomathematics and how can it help children in schools? Teaching Children Mathematics, 7(6), 308-310.

EARGED. (2005). PISA 2003 Projesi - Ulusal Nihai Rapor. Ankara: MEB Eğitimi Araştırma ve Geliştirme Dairesi Başkanlığı.

Ernest, P. (1991). The Philosophy of Mathematics Education, Studies in Mathematics Education. London: Routledge Falmer.

Fasheh, M. (1997a). Mathematics, culture and authority. In A.B. Powell, \& M. Frankenstein (Eds.), Ethnomathematics: Challenging Eurocentrism in mathematics education (pp. 273-290). New York: State University of New York Press.

İzmirli, G.M. (2011). Pedagogy on the Ethnomathematics-Epistemology Nexus: A Manifesto. Journal of Humanistic Mathematics, 1(2), 27-50.

Kaleva, W., Matang, R., \& Owens, K. (2008). PNG Measurement Project. http://www.uog.ac.pg/glec/measurement/MeasurementProject.htm\#_Toc2019 01704 [10.02.16]. 
Kırdar, M. (2009). Explaining Ethnic Disparities in School Enrollment in Turkey. Economic Development and Cultural Change, 57(2), 297-333.

Lipka, J., Hogan, M.P., Webster, J.P., Yanez, E., Adams, B., Clark, S., \& Lacy, D. (2005). Math in a cultural context: Two case studies of a successful culturally based math project. Anthropology and Education Quarterly, 36(4), 367-385.

Lubienski, S.T. (2001). A second look at mathematics achievement gaps: Intersections race, class and gender in NAEP data. Paper presented at the annual meeting of the American Educational Research association, Seattle, WA.

Masingila, J. (1993). Connecting the Ethnomathematics of Carpet Layerswith School Learning. International Study Group on Ethnomathematics Newsletter, 8(2), 4- 7.

Meaney, T. (2002). Symbiosis or Cultural Clash? Indigenous students learning mathematics. Journal of Intercultural Studies, 23(2), $167-187$.

Merriam, S.B. (2002). Qualitative Research in Practice. San Francisco, CA: JosseyBass.

Miles, M.B., \& Huberman, A.M. (1994) Qualitative data analysis: an expanded sourcebook (2nd ed.). California: Sage.

Moses, R.P. (1994). Remarks on the struggle for citizenship and math/science literacy. Journal of Mathematical Behaviour, $13,107-111$.

Oakes, J. (1990). Multiplying inequalities: The effects of race, social class, and tracking on opportunities to learn mathematics and science. Santa Monica, CA: The Rand Corporation.

Oliveras, M.L. (1996). Etnomatemáticas, formación de profesores e innovación curricular. Granada: Comares.

Oliveras, M.L. (2006). Etnomatemáticas. De la multiculturalidad al mestizaje. En: Matemáticas e interculturalidad, Biblioteca de UNO. 232, 117149. Barcelona: Graó

Owens, K., \& Kaleva, W. (2007). Changing our perspective on measurement: A cultural case study. In J. Watson \& K. Beswick (Eds.), Proceedings of the 30th annual conference of the Mathematics Education Research Group of Australasia (pp.563-573). Sydney: MERGA.

Pinxten, R. (1992). Knowledge as a Cultural Phenomenon. International Journal for the Study of Processes and Temporality of Culture, 5,83-104.

Pinxten, R., \& François, K. (2007). Ethnomathematics in Practice. In K. François, \& J.P. Van Bendegem (Eds.), Philosophical Dimensions in Mathematics Education (pp. 213-227). New York: Springer.

Presmeg, N.C. (1998). Ethnomathematics in Teacher Education. Journal of Mathematics Teacher Education, 1, 317-319.

Sahin, I., \& Gulmez, Y. (2000). Social Sources of Failure in Education: The case in East and Southeast Turkey. Social Indicators Research,49, 83-113.

Schultes, C.N., \& Shannon, K.M. (1997). Mathematics and culture: A unique liberal arts experience. PRIMUS: Problems, Resources, and Issues in Mathematics Undergraduate Studies, 7(3), 222-234.

Secada, W.G. (1992). Race, ethnicity, social class, language, and achievement in mathematics. In D. A. Grouws (Ed.), Handbook of research on mathematics teaching and learning. New York: Macmillan.

Tan-Sisman, G., \& Aksu, M. (2009). Seventh grade students ${ }^{\text {ee }}$ success on the topics of area and perimeter. Illköğretim-Online, 8(1), $243-253$.

Tate, W.F. (1997). Race-Ethnicity, SES, gender, and language proficiency trends in mathematics achievement: An update. Journal for Research in Mathematics Education, 28(6), 652-679.

Civil, M. (2002). Culture and Mathematics: a community approach. Journal of Intercultural Studies, 23(2), 133-148.

Tıraş, S. (1999). Öğrenme-Öğretme Açısından Matematik Öğretmenlerinin Yeterliliği ve Etkili Olma Düzeyleri. D.E.Ü. Buca Eğitim Fakültesi Dergisi, Özel Sayı.

Vithal, R., \& Skovsmose, O. (1997). The end of innocence: A critique of "ethnomathematics". Educational Studies in Mathematics, 34, $131-157$.

Yıldırım, A., \& Şimşek, H. (2003). Sosyal Bilimlerde Nitel Araştırma Yöntemleri. Ankara: Seçkin Yayınları.

Zacharos, K. (2006). Prevailing Educational Practices for Area Measurement and Students' Failure in Measuring Areas. Journal of Mathematical Behavior, 25(3), 224-239. 
ANEXOS

ANEXO 1: Hoja de tarea 4: Calcular el área circundante del olivo

\section{Ara tarımı}

Meyve bahçelerinin ilk yıllarında sıra aralarındaki boş alanlarda yapılan yetiştiriciliğe "ara tarımı" denilmektedir. Ara ürün sadece sulama suyunun bol olduğu yerlerde, geçici bir dōnem için yapılabilir. Ara ūrūn, bahçenin normal sulama ve ilaçlama programına uygun olmalıdır. Yine ara ūrūn zararı, bōcek, virüs, yabancı otlar ve hastalıklara yataklık yapmamalıdır. Ara ủrün ekonomik bakımdan gerekli olabilir. Ancak ağaç geliștikçe ara ürūn alanı daraltılır ve sonuçta kaldırılır. Aksi durumda ağaçların geliş̧mesini būyük ölçüde yavaşlamaktadır.

Zeytin bahçelerinde, hastalık bulaşma riskini artırdığı için Badem, Kayısı, Kiraz,

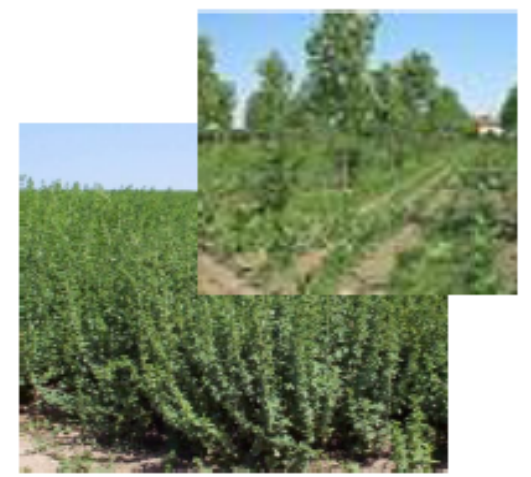
Erik, Şeftali, Asma, Akçaağaç, At kestanesi, Karaağaç, Böğürtlen, Karpuz, Çilek, Pamuk, Bamya, Domates, Biber, Patlıcan, Patates, Ayçiçeği gibi bitkilerin ara tarımı yapılmamalıdır.

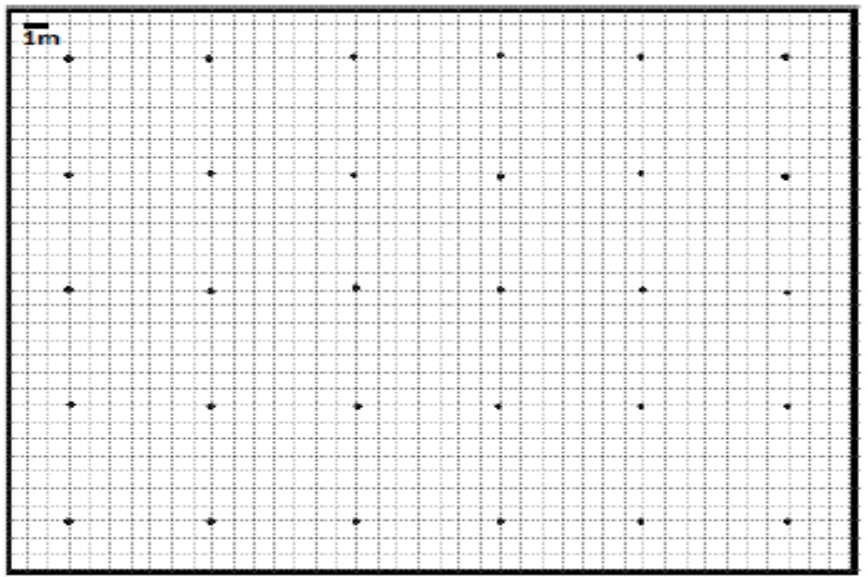

Sckilde krokisi verilen aroziye kare şeklinde bodur zeytinler dikilmiştir. Dar taçlı ve kısa boylu olduğu için yaşam alanı sadece $4 \times 4 \mathrm{~m}$ $=16 \mathrm{~m}^{2}$ olan bodur zeytinden arta kalan boș arazi, 7 yıl verim alabilecek şekilde kekık ekılerek degerlendırılmek ıstenıyor. Buna göre, kekik ekmek için kaç $\mathrm{m}^{2}$ arazimiz olduğunu hesaplayınız. 


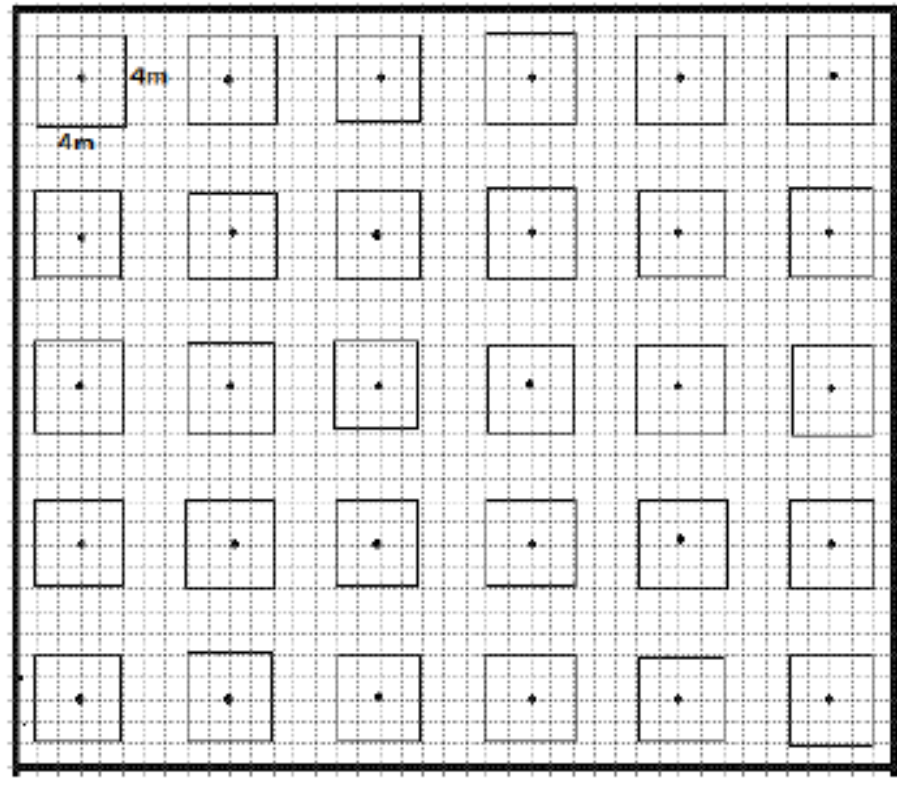

$\checkmark$ Ağaçlann yaşam alanı șekil üzerinde gōsteriniz:

$\checkmark$ Bütün alan bulunuz:

$\checkmark$ Bütün alandan, ağaçların yaşam alanı çıkarılarak gerıye kalan arazı mıktannı hesaplayını:

ANEXO 2: Hojas de tarea 6: El arbol de la vida; la relación entre la longitud del lado del cuadrado y el área
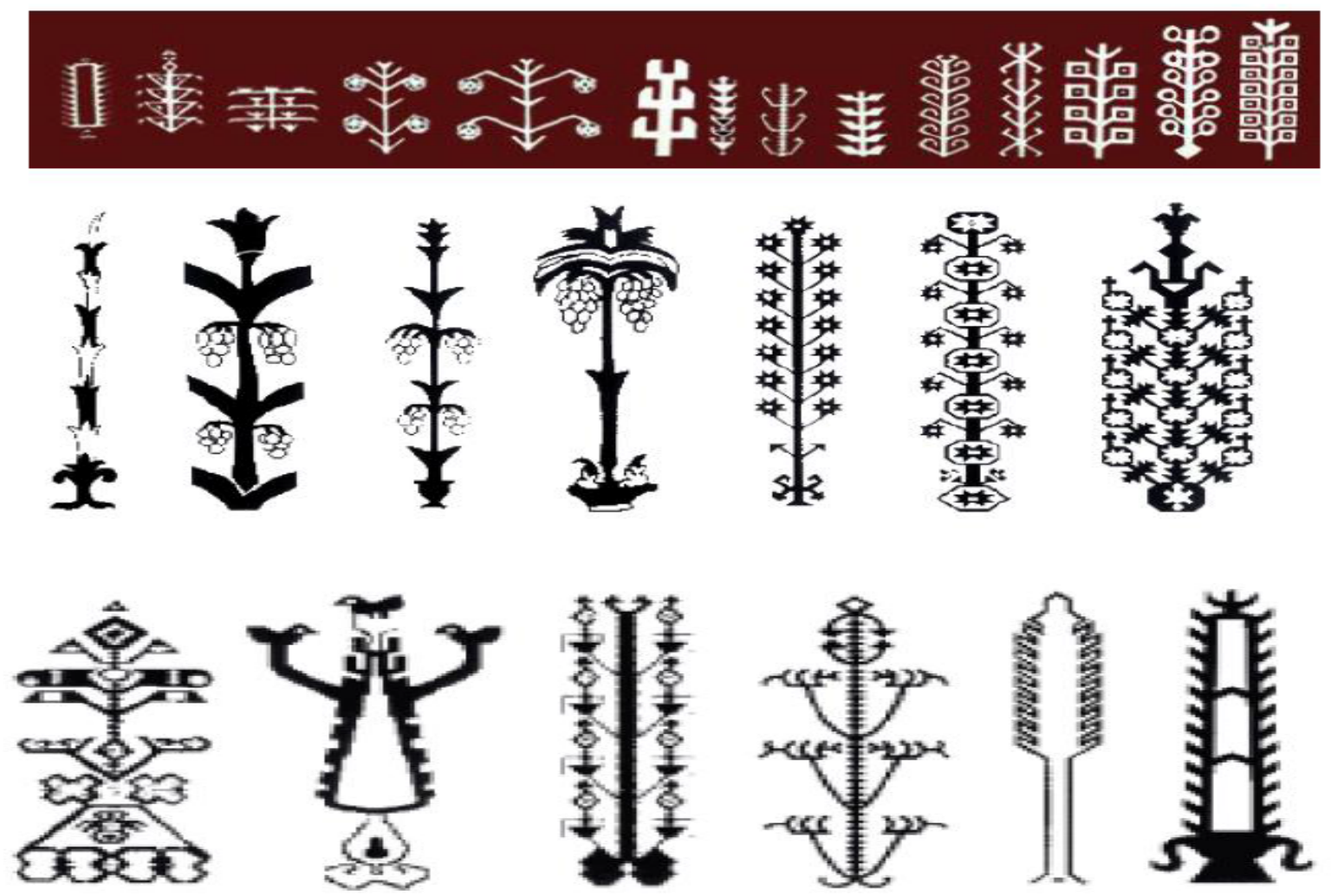
HAYAT ĂĞAI, kökeni tarih-öncesi denilen devirlere kadar uzanan, bașta Asya şamanistleri olmak üzere, pek çok gelenekte rastlanan bir semboldür.

Eski Türk geleneg̃ine ve Şaman inançlarına göre, bu Dünya'yı ortasından (göbeğinden) öte-âleme ve Demir-Kazık Yıldızı'na bağlayan, dalları vasıtasıyla şamanlara yeryüzünden yüksek âlemlere yolculuk yapma olanağı sağlayan bir ağaçtır.

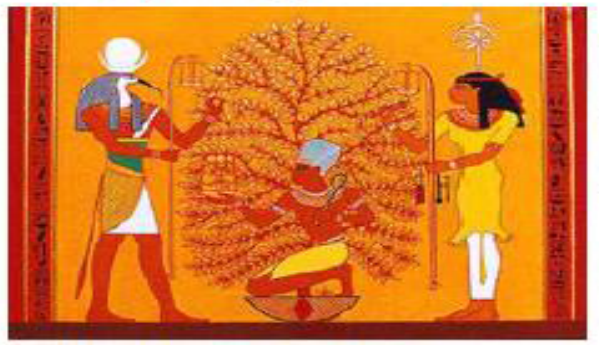

Geniş anlamda sürekli gelişim ve değişim içinde yaşayan evreni sembolize eder. Evrenin üç elementini: toprağın derinliğine

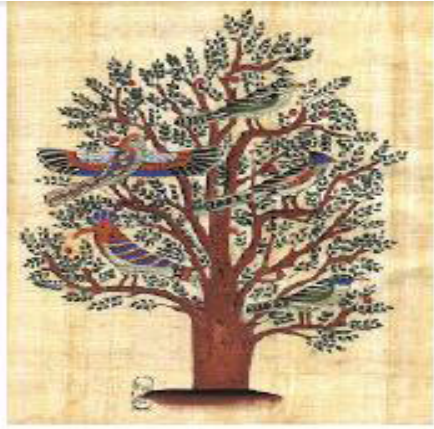
inen kökleriyle yeraltını, alt dalları ve gövdesiyle gökyüzünü, ışı̆a yüselen ust dallarıyla cenneti birleştirir.

İnsanoğlu geçmişten gūnümüze uzanan yolculuğunda pek çok varlığı kutsal saymış, kutsal

Eski Mıst' 'dan bir Hayat Ağaca örneḡi saydığı varlıklari yazıya, dile, dine, sanata dökerek günümüze ulaşmasını sağlamıştır. İnsanoğlu'nun bu kutsal saydığı varoluşlardan belki de en bilineni Hayat Ağacı'dır.

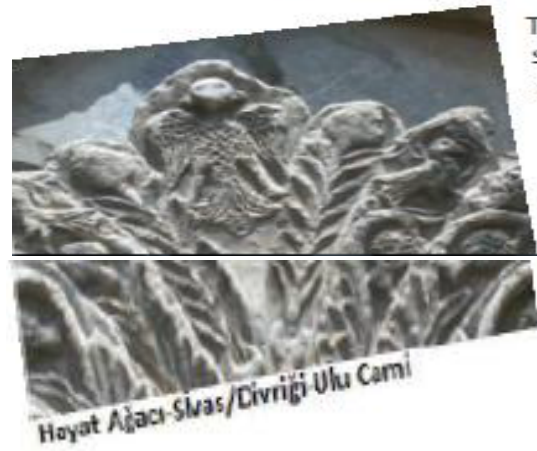

Toplumlarda hayat ağacının sembollerinden bir tanesi de zeytin ağacıdır. Bazı yazılı kaynaklarda tarif edilen yaşam ağacı figürü zeytin ağacina oldukç̧a benzemektedir: Yakut geleneğinde, Yerin

Göbeği'nden çkan, çiçek açan bu ağacın tepe kısmının köpüklū, sarı,

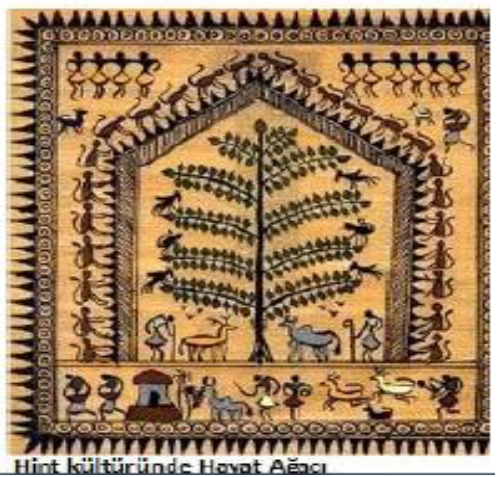
insanlara şifa verici bir sıvı içinde olduğu ifade edilir. Aynca Hıristiyan geleneğine göre, yaşam ağacı,12 defa meyve veren, yaprakları ulusların şifa bulmasını sağlayıcı bir ağaç olarak belirtilir( Vahiy, 22/2).

Hayat Ağacı formu, birçok uygarlığın çeşitli stilize motiflerle taş, tahta, çömlek, çini işleme, dokuma, cam, tezhip, minyatür, edebiyat ve müziğinde yer almışır.

Sonuç olarak hayat ağacı hemen hemen bütün kültürlerde bir şekilde var olmuş ve önemli sayılmıştır. Var olduğu ilk dönemlerden, modern dönemlere kadar kullanımını gördüğümüz

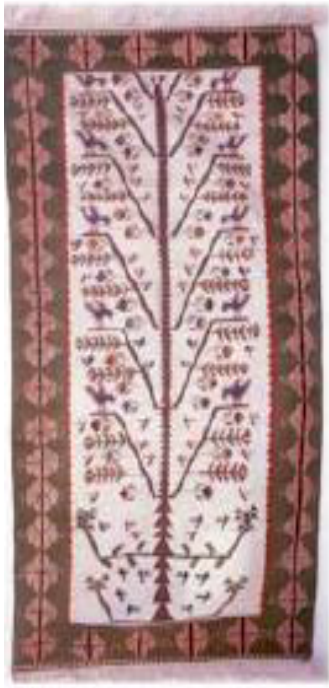
hayatag̈ac, bugün otantik figürler olarak karşımıza çikmaktadir. Anadolu'da önemi hep var olan hayatağacı Türk- İslam sanatında da pek çok şekilde yer almakta ve el sanatlarından, inançlara kadar her yerde karşımıza çikmaktadir.

Sonsuzluğun sembolü, ölümsüzlüğü araştırmanın ve ölümden sonra yaşam olduğu umudunun bir nişanı olan hayat ağac motifi, geleneksel Anadolu işleme sanatlarında en çok kullanılan motiflerden biri olagelmiştir.

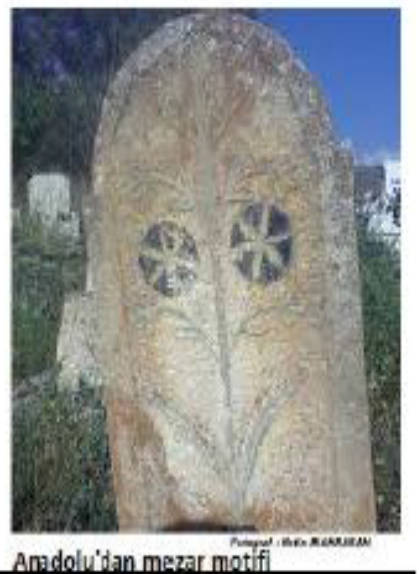




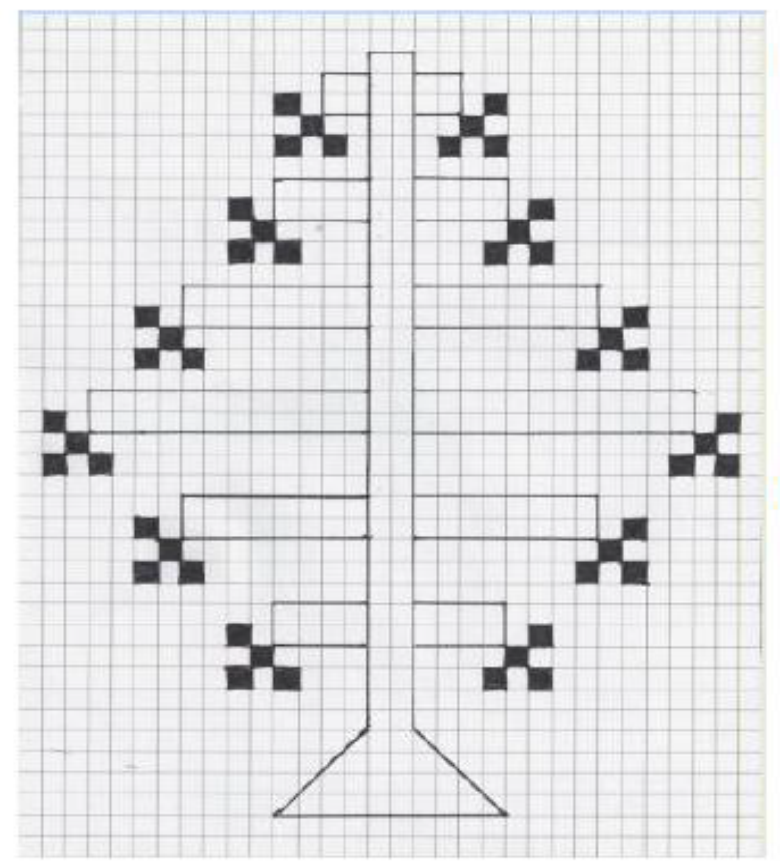

a) Şekilde verilen hayat ağacı motifinin bütün kenar uzunlukları iki katına çıkarılarak yastık üzerine işlenecektir. Oluşan yeni motifin alanının kaç birim kareyi kapladığını hesaplayınız. Yeni motif ile orijinalinin alanlarını kıyaslayınız.

Normalde 100 ilmek ile işlenen bu motifin kenar uzunlukları 3 katına çıkarılınca ne kadar ilmek atılması gerekir?

b) Şekilde verilen hayat ağacı motifinin bütün kenar uzunlukları yarıya indirilerek çarşaf kenarlarına ișlenecektir. Oluşan yeni motifin alanının kaç birim kareyi kapladığıı hesaplayını. Yeni motif ile orijinalinin alanlarıı kıyaslayını.

Normalde 100 ilmek ile işlenen bu motifin kenar uzunlukları yarıa indirilince ne kadar ilmek atılması gerekir? 